\title{
DEMONSTRATION OF SAR INTERFEROMETRY UNDER CROSSING ORBITS USING TERRASAR-X AND TANDEM-X
}

\author{
Paco López-Dekker, Pau Prats, Francesco De Zan, Steffen Wollstadt, Daniel Schulze, \\ Gerhard Krieger and Alberto Moreira
}

Microwaves and Radar Institute

German Aerospace Center (DLR)

\begin{abstract}
This paper discusses cross-track SAR interferometry under crossing orbits. The underlaying theory is briefly outlined, showing that a crossing angle between the ground tracks needs to be compensated by applying different squint angles in order to have overlap in the the ground-spectral domain. Two sets of crossing orbits InSAR experiments are described. The results of the first experiment, are discussed.
\end{abstract}

\section{Index Terms - SAR, SAR interferometry}

\section{INTRODUCTION}

SAR Interferometry theory usually considers geometries in which the trajectories corresponding to the pair of InSAR acquisitions are almost perfectly parallel. A consequence of this condition is the common understanding that the two acquisitions most be acquired with similar, if not identical, Doppler centroids $\left(f_{D C}\right)$ in order to maximize the common Doppler spectral content of the images. In the case of InSAR under non-parallel trajectories, however, this common understanding is no longer valid, and an offset of the individual Doppler spectra is necessary in order to achieve a coherent interferogram. Interestingly, this phenomenon was reported in 1988 [1], in the context of the SIR-B experiments.

The required frequency offset of the individual SAR images increases as the angle between the trajectories augments. As a consequence, at some point the common spectral content cannot be obtained just from filtering the available spectra of two acquisitions with the same nominal $f_{D C}$. In this case, it is necessary to acquire the images with a carefully controlled $f_{D C}$ offset, i.e. with a carefully controlled relative squint. The azimuth steering capabilities of TerraSAR-X (TSX) and its sibling TanDEM-X (TDX) satellite (TerraSAR$X$ add on for Digital Elevation Measurements), together with their uniquely flexible commanding, turns them into an ideal platform for experiment with these type of non-parallel acquisitions.

The TanDEM-X project is partly funded by the German Federal Ministry for Economics and Technology (Förderkennzeichen $50 \mathrm{EE} \mathrm{1035)}$
Section 2 of this papers briefly presents a reformulation of the crossing-orbit interferometry problem in terms of a 2 D spectral rotation of the sampled ground spectrum. Section 3 describes a set of experimental acquisition, while the results are discussed in Section 4.

\section{THEORY}

Let us assume a SAR system that moves along the $\mathrm{x}$-axis and, for simplification, over a flat terrain. The resulting SAR image samples the 2-D spectrum of the complex scattering coefficient $\sigma(x, y)$, so that the resulting image can be expressed, in the wavenumber domain, as

$$
S_{0}\left(k_{x}, k_{y}\right)=\sigma\left(k_{x}, k_{y}\right) W\left(k_{x}-k_{x 0}, k_{y}-k_{y 0}\right),
$$

with $W(\cdot, \cdot)$ some baseband windowing function,

$$
\begin{aligned}
& k_{x 0}=\frac{2 \pi f_{D C_{0}}}{v_{x}}=2 k_{0} \sin \psi_{0} \sin \theta_{0} \\
& k_{y 0}=2 k_{0} \cos \psi_{0} \sin \theta_{0},
\end{aligned}
$$

$y$ represents the ground range direction, $f_{D C_{0}}$ the Doppler Centroid, $v_{x}$ the horizontal velocity of the system, $k_{0}$ the wavenumber associated to the carrier frequency, $\psi_{0}$ is the ground projection of the squint angle, and $\theta_{0}$ is the incidence angle. Basically, this represents a rectangular spectrum centered at $\left(k_{x 0}, k_{y 0}\right)$.

Let us now consider a second image acquired by a system flying in a slightly different direction, rotated by an angle $\alpha$. In its own coordinate system, the spectrum of this second image will be

$$
S_{1}\left(k_{x}^{\prime}, k_{y}^{\prime}\right)=\sigma\left(k_{x}^{\prime}, k_{y}^{\prime}\right) W\left(k_{x}^{\prime}-k_{x 1}^{\prime}, k_{y}^{\prime}-k_{y 1}^{\prime}\right),
$$

which again will correspond to a rectangular spectrum centered this time at $\left(k_{x 1}^{\prime}, k_{y 1}^{\prime}\right)$. Considering the reference system rotation, the 2-D spectrum of the second image in the original reference system is centered at

$$
\begin{aligned}
& k_{x 1}=k_{x 1}^{\prime} \cos \alpha-k_{y 1}^{\prime} \sin \alpha \\
& k_{y 1}=k_{x 1}^{\prime} \sin \alpha+k_{y 1}^{\prime} \cos \alpha .
\end{aligned}
$$


and, the entire spectrum can be written as

$$
S_{1}\left(k_{x}, k_{y}\right)=\sigma\left(k_{x}, k_{y}\right) W^{\prime}\left(k_{x}-k_{x 1}, k_{y}-k_{y 1}\right),
$$

where $W^{\prime}(\cdot, \cdot)$ represents the squinted spectral window. The two spectra are conceptually represented in the left diagram of Figure 1 for the case in which both images are acquired with zero Doppler Centroid (DC). In order to be able to form a useful interferogram it is clear that the overlap between the spectra given by (1) and (5) should be maximized. It is now useful to define

$$
\begin{aligned}
& \Delta k_{x}=k_{x 1}-k_{x 0} \\
& \Delta k_{y}=k_{y 1}-k_{y 0} .
\end{aligned}
$$

There will be spectral overlap if these two terms are small compared to $2 \pi / \delta x$ and $2 \pi / \delta y$, respectively, where $\delta y$ represents the ground range resolution and $\delta x$ the azimuth resolution. For small values of $\alpha$, similar incidence angles $(\Delta \theta=$ $\left.\theta_{1}-\theta_{0} \approx 0\right)$, and small squint angle difference $(\Delta \Psi=$ $\left.\Psi_{1}-\Psi_{0}\right)$ these spectral shifts reduce to:

$$
\begin{aligned}
& \Delta k_{x} \approx 2 k_{0}\left(\sin \psi_{0} \cos \theta_{0} \Delta \theta+\cos \psi_{0} \sin \theta_{0}(\Delta \psi-\alpha)\right) \\
& \Delta k_{y} \approx 2 k_{0}\left(\cos \psi_{0} \cos \theta_{0} \Delta \theta-\sin \psi_{0} \sin \theta_{0}(\Delta \psi-\alpha)\right) .
\end{aligned}
$$

Assuming also small squints (or Doppler Centroids) yields

$$
\begin{aligned}
\Delta k_{x} & \approx 2 k_{0} \sin \theta_{0}(\Delta \psi-\alpha)=\frac{2 \pi \Delta f_{D C}}{v_{x}}-2 k_{0} \alpha \sin \theta_{0} \\
\Delta k_{y} & \approx 2 k_{0} \cos \theta_{0} \Delta \theta
\end{aligned}
$$

The range spectral shift, $\Delta k_{y}$, reduces to the typical spectral shift studied for parallel orbits [2]. Note that a variation of the incidence angle given by $\Delta \theta$ is the result of a normal baseline component. The azimuth spectral shift, $\Delta k_{x}$, depends on the angle between the orbits and on the relative squint between the two acquisitions. It is zero if the horizontal rotation of the reference system $(\alpha)$ equals the ground projection of the squint angle $(\Delta \psi)$. Geometrically this means that the target is being observed from the same ground projected direction.

The alignment of the spectra after applying a relative squint is illustrated by the right diagram in Figure 1, where all the angles are clearly exaggerated, and a zero cross-track baseline is assumed. In practice for small rotations and squint angles, and considering that $k_{y 0} \gg 2 \pi / \delta_{y}$, their effect is practically reduced to a spectral shift in azimuth.

A pair of images acquired with different squints in order to compensate the crossing angle will present different Doppler spectra in their respective range-azimuth coordinates. It is interesting to note, however, that the spectral components will be automatically aligned by the interferometric processing, namely, the flat earth phase removal. Hence, any common-band filtering in azimuth should be done taking into account the spectral modulation.

\begin{tabular}{|c|c|c|c|}
\hline & Predicted & Configured & Real \\
\hline$\Delta k_{x}(\mathrm{rad} / \mathrm{m})$ & -0.92 & - & -0.93 \\
$\Delta k_{y}(\mathrm{rad} / \mathrm{m})$ & -0.81 & - & -0.825 \\
Relative squint & $0.126^{\circ}$ & $0.145^{\circ}$ & $0.127^{\circ}$ \\
\hline
\end{tabular}

Table 1: Crossing orbit InSAR parameters for first experiment

\section{EXPERIMENT DESCRIPTION}

Two sets of experiments have been conducted.

\subsection{Pursuit Monostatic acquisitions}

During the weeks previous to this first stable interferometric configuration, reached on July 20, TDX had to carefully approach TSX from an initial along-track separation of 16000 $\mathrm{km}$. For two spacecraft placed in the same orbital plane, an along-track separation results in different ground-tracks due to Earth's rotation. The ground-tracks have a maximum separation at the equator but cross at some point at high latitudes.

The data considered in this paper corresponds to a descending acquisition over October Revolution Island, in the Russian Arctic, on July 16th at 00:41 UTC. Based on the predicted TSX and TDX orbits, the expected azimuth spectral shift was $-0.92 \mathrm{rad} / \mathrm{m}$ at 40 degree off-nadir look angle, requiring a DC offset of $1.1 \mathrm{kHz}$, which is a significant fraction of the Doppler Bandwidth. The desired $\Delta f_{D C}$ corresponds to a relative azimuth antenna squint of $0.13^{\circ}$, with the trailing TDX system looking forward with respect to TSX. In practice, TSX was configured with a backward squint of $-0.066^{\circ}$, and TDX with a forward squint of $0.079^{\circ}$, resulting in a relative squint of $0.145^{\circ}$ (see Table 1).

The cross-track baseline varied during the $29 \mathrm{~s}$ acquisition (200 km strip image) from $1.9 \mathrm{~km}$ to $2.4 \mathrm{~km}$. For the results presented below it was approximately $2.0 \mathrm{~km}$, which corresponds to a height of ambiguity of $3.8 \mathrm{~m}$. This is about an order of magnitude smaller than the nominal values assumed for the mission (in bistatic operations).

\subsection{Quasi-repeat pass acquisitions}

The same acquisition concept can be applied for a single spacecraft considering different tracks. Since the orbital plane is more or less fixed in an inertial frame, tracks separated in time by approximately a whole number of days tend to be relatively close. For the 11 day repeat-cycle orbit of TSX, the closest pairs of tracks correspond to a temporal lag of 5 or 6 days, and the second closest pairs to a 1-day lag. As before, it is only possible to obtain small enough cross tracks at very high latitudes. Moreover, since the angle between the ground-tracks is much larger the region where the 
Zero-Doppler Acquisitions

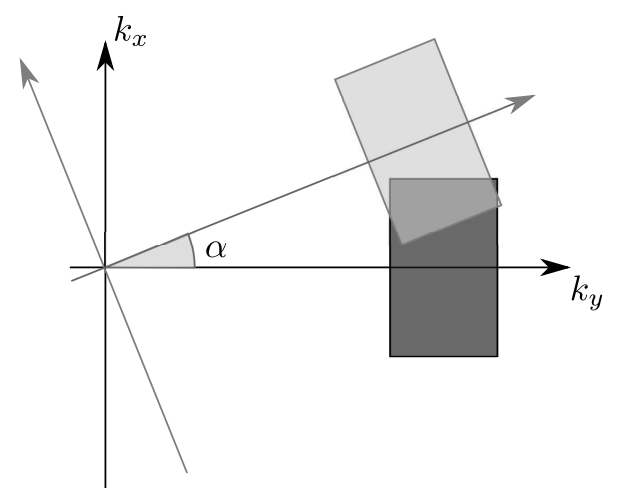

Squinted Acquisitions

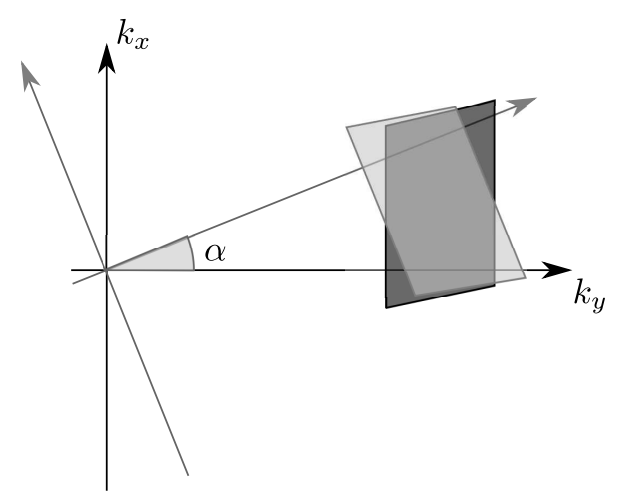

Fig. 1: Conceptual representation of the 2-D ground spectra for a pair of crossing acquisitions. Due to the rotation, if both images are acquired with $f_{D C}=0$, different parts of the 2-D ground spectrum are sampled. By acquiring the data with some relative squint, the spectral overlap is maximized.

\begin{tabular}{|c|c|c|c|c|}
\hline$\Delta \mathrm{t}$ (days) & $\Delta k_{x}$ & $\Delta k_{y}$ & Required $\Delta$ squint & $\mathrm{h}_{a m b}(\mathrm{~m})$ \\
\hline 1 & -21.8 & -0.29 & $2.96^{\circ}$ & 8.0 \\
5 & 10.9 & -0.07 & $-1.48^{\circ}$ & 41.9 \\
\hline
\end{tabular}

Table 2: Crossing orbit InSAR spectral shifts (before squinting) and required relative squint for second experiment

baselines are adequate for interferometry are constrained to a very narrow range of latitudes.

As an experimental site, an area located approximately at $78^{\circ} \mathrm{S}, 57^{\circ} \mathrm{W}$, corresponding to the Antarctic ice cap, was chosen. The location was selected with the goal of minimizing the range spectral shifts for both a 1 day and a 5 day quasirepeat pass acquisitions, as shown Table 2. A set of three data takes on April 20th, 25th and 26th of 2011, so that there was a 5 day lag between the first and second acquisition, and 1 days between the second and the third, and then repeated in the next 11 day cycle. In order to minimize the negative impact of spectral shift in range, a $300 \mathrm{MHz}$ pulse-bandwidth was used and an incidence angle of $47^{\circ}$ was selected. The required relative squint angles were of $0.039^{\circ},-1.447^{\circ}$ and $1.526^{\circ}$, respectively.

\section{RESULTS}

The results shown in Figure 2 correspond to a $20 \mathrm{~km}$ groundrange by $31 \mathrm{~km}$ in azimuth strip of October Revolution Island. The slant-range-azimuth images show, from left to right, the relative brightness, the interferometric phase and the interferometric coherence. The images have been rotated so that North is roughly at the top. A 9x6 multi-look window has been applied to the data yielding $12 \times 12 \mathrm{~m}^{2}$ pixels. SAR focusing and interferometric processing was performed using the Microwaves and Radar Institute's Experimental Interferometric processor (TAXI) [3]. The data were processed without any azimuth common-band spectral filtering. In range, the spectral shift was in the order of $20 \%$ of the available bandwidth, making common-band filtering a necessity. The high interferometric coherence obtained, up to 0.95 in some areas, illustrates how the originally displaced Doppler spectra is aligned after co-registation and flat earth removal.

Figure 3 shows a rendering of the DEM obtained, with height ranging from $0 \mathrm{~m}$ at sea-level to $585 \mathrm{~m}$ at the SouthWestern corner of the image. With the estimated coherence, the standard deviation of the point-to-point errors is, in most cases, in the $10-20 \mathrm{~cm}$ range, with values down to $5-6 \mathrm{~cm}$ in the high coherence areas.

\section{CONCLUSION}

The theory and results discussed in this paper show the possibility of acquiring compatible interferometric pairs with a significant squint angle between the ground tracks of the orbits. Somewhat counter-intuitively, it is shown that under these conditions, the images must be acquired with relative squint angles leading to significant large Doppler Centroids (the Doppler Centroid difference can, in fact be much larger than the PRF).

The first experiment resulted in an unusually large interferometric baseline. The results obtained show that, due to the high coherence inherent to single-pass interferometry, a formation flying InSAR mission can result in height accuracies in the order of $10 \mathrm{~cm}$. This may be exploited in the future, for example, to measure changes of (fast decorrelating) ice topography.

Future work will focus on processing and acquiring more quasi-repeat pass experiments. The resulting data sets, offering 1,5 and 11 day repeat pass intervals, may be interesting, 


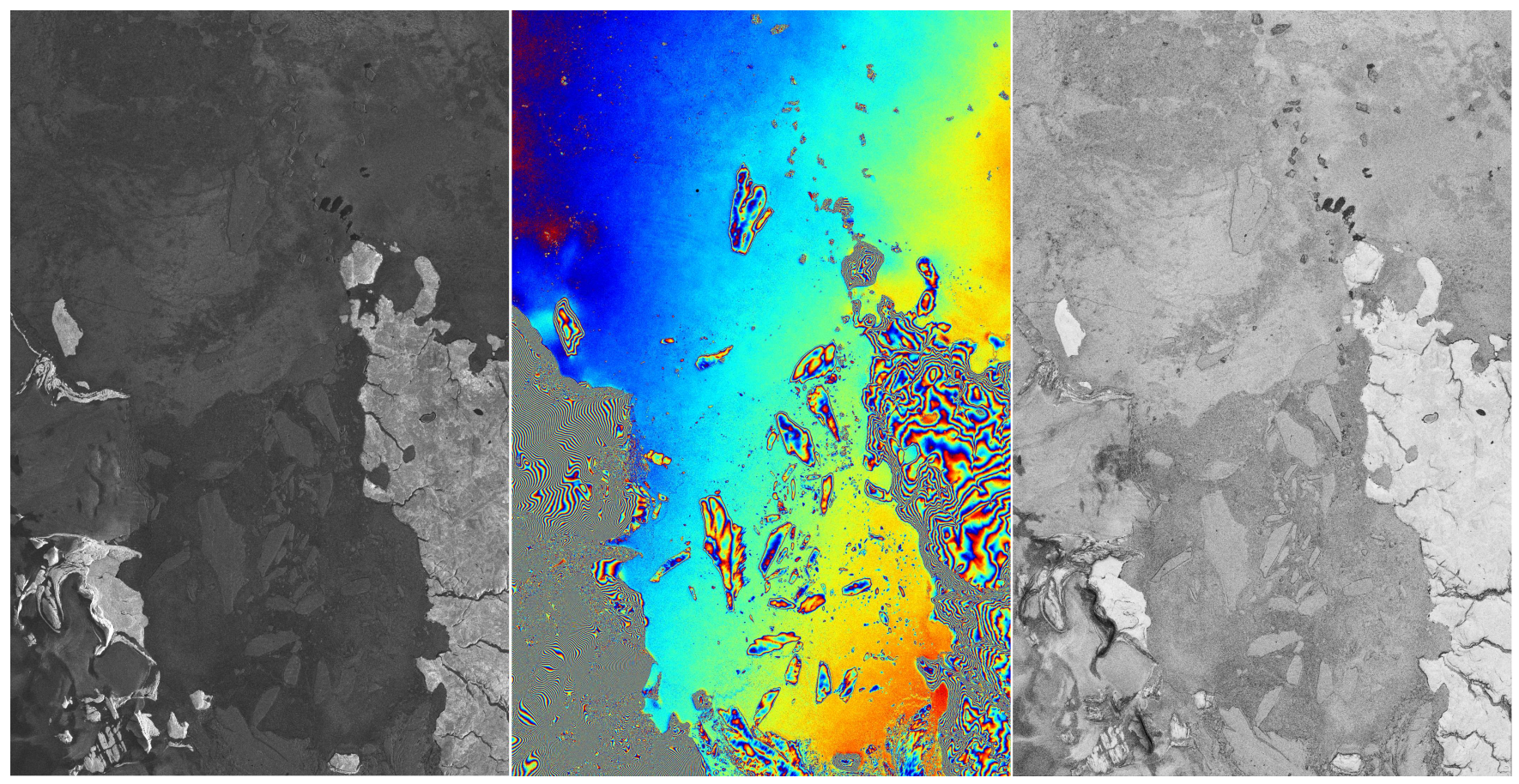

Fig. 2: From left to right: relative brightness, interferometric phase, and interferometric coherence of an area corresponding to the North-Eastern coast of October Revolution Island, in the Russian Arctic. The images are in slant-range (from right to left) azimuth (top to bottom) coordinates.

to study the temporal decorrelation behavior of polar ice.

\section{REFERENCES}

[1] Andrew K. Gabriel and Richard M. Goldstein, "Crossed orbit interferometry: theory and experimental results from SIR-B," International Journal of Remote Sensing, vol. 9, no. 5, pp. 857, 1988.

[2] F. Gatelli, A.M. Guarnieri, F. Parizzi, P. Pasquali, C. Prati, and F. Rocca, "The wavenumber shift in SAR interferometry," Geoscience and Remote Sensing, IEEE Transactions on, vol. 32, no. 4, pp. 855-865, 1994.

[3] Pau Prats, Marc Rodriguez-Cassola, Luca Marotti, Matteo Nannini, Gerhard Krieger, and Andreas Reigber, "TAXI: a versatile processing chain for experimental TanDEM-X product evaluation," in Proceedings of the International Geoscience and Remote Sensing Symposium, Honolulu, 2010.

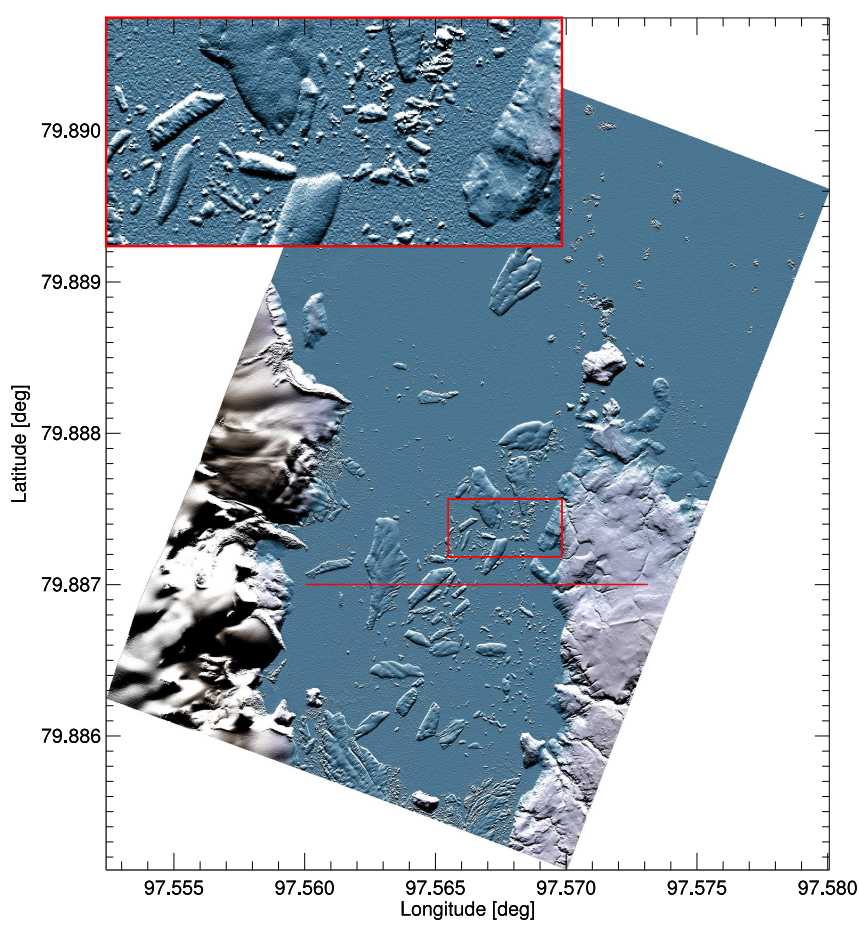

Fig. 3: Geocoded and rendered DEM. The zoom-in (red rectangle) illustrates the high level of topographic detail. 